MRS Advances (C) 2017 Materials Research Society

DOI: $10.1557 /$ adv.2017.280

\title{
Choice of Polymer Matrix for a Fast Switchable III-V Nanowire Terahertz Modulator
}

\author{
Sarwat A. Baig ${ }^{1}$, Jessica L. Boland ${ }^{2}$, Djamshid A. Damry ${ }^{2}$, Hoe H Tan ${ }^{3}$, Chennupati Jagadish ${ }^{3}$, \\ Michael B. Johnston ${ }^{2}$ and Hannah J Joyce ${ }^{1}$ \\ ${ }^{1}$ Department of Engineering, University of Cambridge, Electrical Engineering Building, $9 \mathrm{JJ}$ \\ Thomson Avenue, Cambridge, CB3 0FA, UK \\ ${ }^{2}$ Department of Physics, University of Oxford, Clarendon Laboratory, Parks Road, Oxford OX1 \\ 3PU, UK. \\ ${ }^{3}$ Department of Electronic Materials Engineering, Research School of Physics and Engineering, \\ The Australian National University, Canberra, ACT 0200, Australia
}

\begin{abstract}
Progress in ultrafast terahertz $(\mathrm{THz})$ communications has been limited due to the lack of picosecond switchable modulators with sufficient modulation depth. Gallium arsenide nanowires are ideal candidates for $\mathrm{THz}$ modulators as they absorb $\mathrm{THz}$ radiation, only when photoexcited giving the potential for picosecend speed switching and high modulation depth. By embedding the nanowires in a polymer matrix and laminating together several nanowire-polymer films, we increase the areal density of nanowires, resulting in greater modulation of $\mathrm{THz}$ radiation. In this paper, we compare PDMS and Parylene C polymers for nanowire encapsulation and show that a high modulation depth is possible using Parylene $\mathrm{C}$ due to its thinness and its ability to be laminated. We characterize the modulator behavior and switching speed using optical pump$\mathrm{THz}$ probe spectroscopy, and demonstrate a parylene-nanowire $\mathrm{THz}$ modulator with $13.5 \%$ modulation depth and 1ps switching speed.
\end{abstract}

\section{INTRODUCTION}

The terahertz $(\mathrm{THz})$ frequency range is located between the infrared and microwave regions of the electromagnetic spectrum $(0.1$ to $10 \mathrm{THz})[1]$. Whilst huge advances have been made in $\mathrm{THz}$ generation[2] and detection[3], to our knowledge, an ultrafast switchable $\mathrm{THz}$ modulator has not yet been demonstrated. This is crucial in the development of high speed $\mathrm{THz}$ communications as components capable of encoding information into the $\mathrm{THz}$ waveform are essential[4]. This THz modulator needs (1) a high modulation depth, (2) picosecond switching speed, (3) to be electrically or optically controllable, (4) to be easily integrated into current silicon-based technology[5]. High performance modulators in the field have either demonstrated a high modulation depth or slower switching times (graphene)[6] - or a low modulation depth, but fast (ps) switching time (carbon nanotubes)[7].

III-V nanowires (NWs) are of particular interest for use in THz applications due to their high electron mobilities[8] and opportunity for integration with silicon devices[9]. Gallium arsenide (GaAs) NWs are ideal candidates for THz modulation, as they exhibit a direct band gap and short carrier lifetime[10]. When GaAs NWs are photoexcited, free charge carriers are generated in NWs whose long axes are aligned with the pump beam polarization. These charge carriers are able to interact with THz electric field. In Figure 1a(i), the Thz pulse is attenuated when the pump polarization is parallel to the long axis of the NW and is transmitted when the pump polarization is aligned perpendicular to the NW ( Figure 1a(ii)). Therefore GaAs NWs are ideal candidates to be used as the transient $\mathrm{THz}$ absorber in ultrafast $\mathrm{THz}$ modulators.[11] 


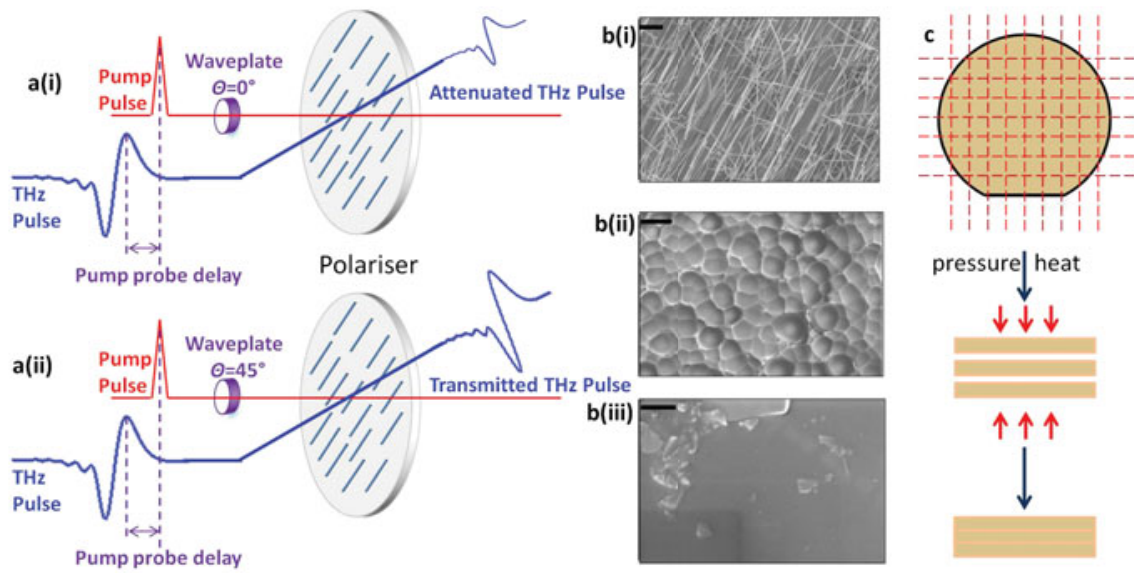

Figure 1. (a-i) Photoexcitation generates free carriers when the pump polarization is aligned parallel to the long axis of the NWs. This results in attenuation of THz pulse. (a-ii) When the pump is aligned perpendicular to the NW, the THz is transmitted. (b) SEM images of (i) aligned GaAs NWs and NWs coated with (ii) Parylene C and (iii) PDMS. Scale bar shows $2 \mu \mathrm{m}$ in all images. (c) Schematic showing how multilayered modulators were fabricated.

The NWs need to be embedded into a polymer matrix to be used in devices. This allows them to be removed from the substrate, whilst still maintaining parallel alignment. We highlight Parylene C and PDMS (polydimethyl siloxane). Both are robust, flexible and encapsulate the nanowires well, and allow for simple thin film lift off [12] [13]. In addition Parylene C and PDMS are transparent to $\mathrm{THz}$ radiation (refractive indices of 1.6 and 1.4 respectively). Any transient $\mathrm{THz}$ absorption observed is solely due to the photoexcited NWs.

Here we demonstrate the fabrication and characterization of an ultrafast $\mathrm{THz}$ modulator based on aligned GaAs NWs embedded in Parylene C and PDMS. First we discuss modulator fabrication and the suitability of the two polymer matrices. We then characterize the modulator performance and properties using optical pump-THz probe (OPTP) spectroscopy.

\section{EXPERIMENT}

\section{Nanowire growth, polymer deposition and modulator fabrication}

GaAs nanowires (diameter $50 \mathrm{~nm}$ ) were grown via a gold-seeded metal-organic chemical vapour deposition in the $<111 \mathrm{~B}>$ direction at an angle of $35.3^{\circ}$ to the $\mathrm{GaAs}<100>$ substrate. Figure 1b(i) shows a plan-view scanning electron microscope (SEM) image of these well aligned NWs. Prior to polymer deposition, the NW wafers were checked under SEM and the general NW alignment was marked on the back of the wafer. The wafers were then coated: one with a $5 \mu \mathrm{m}$ layer of Parylene C (Gorham process, PSD 2010 LabCoter2), the other with a $300 \mu \mathrm{m}$ layer of PDMS: Sylgard 184 (mixed 10:1 base:curing agent ratio), spin coated at $250 \mathrm{rpm}$, then baked at $150^{\circ} \mathrm{C}$. Figure $1 \mathrm{~b}$ (ii) and $1 \mathrm{~b}$ (iii) shows SEM images of the NWs coated with Parylene $\mathrm{C}$ and PDMS respectively. As shown in Figure 1c, the polymer layers were divided into equal sized 
segments using a razor blade to form $10 \mathrm{~mm}$ x $10 \mathrm{~mm}$ squares and were peeled off from the substrate to create a free standing polymer-nanowire thin films. The films were stacked carefully, ensuring the nanowire alignment was consistent all the way through the layers. The Parylene $\mathrm{C}$ thin films were hot-bonded together at $120^{\circ} \mathrm{C}$ and 20 bar using an Obducat nanoimprinter, whilst maintaining the alignment of the nanowires.[14]. Laminated PDMS thin films were not amenable to hot bonding and instead adhesion between layers was achieved via the inherent stickiness of the PDMS.

\section{Optical pump-THz probe (OPTP) spectroscopy measurements}

A Ti:sapphire regenerative amplifier laser with pulse duration of $35 \mathrm{fs}, 5 \mathrm{kHz}$ repetition rate and centre wavelength of $800 \mathrm{~nm}(\mathrm{~h} v=1.55 \mathrm{eV})$ was used[7]. The laser pulse was divided into three beams: (1) photoexcites the modulator (beam width: $5 \mathrm{~mm}$, fluence maximum of 280 $\mu \mathrm{J} \mathrm{cm}^{-2}$ ); (2) generates the terahertz probe beam by optical rectification in a $2 \mathrm{~mm}<110>$ (gallium phosphide) GaP crystal, (THz beam width: $0.48 \mathrm{~mm}$ at the nanowire film); (3) acts as a gate beam for electro-optical sampling of the THz field via a $200 \mu \mathrm{m}<110>\mathrm{GaP}$ crystal. The transmission of the electric field of the THz probe pulse through the nanowires, $E$, was measured as a function of time. The value of $\Delta E / E_{\text {off }}$ is measured with respect to pump polarization and time to extract modulation depth and photoexcitation lifetime respectively. We define $t=0$ to be the pump-probe delay at which $1-\Delta E / E_{\text {off }}$ is minimum.

\section{DISCUSSION}

On fabricating the Parylene C and PDMS thin films, we evaluated the lifetime of the photoexcited carriers in the GaAs NWs using OPTP. Single layers of PDMS and Parylene C coated GaAs NW modulators were measured. The normalized THz transmission $1-\Delta E / E_{\text {off }}$ as a function of time $t$ after photoexcitation were measured at 30,60 and $280 \mu \mathrm{J} \mathrm{cm}^{-2}$ pump fluences. The pump polarization and $\mathrm{THz}$ polarization were aligned parallel to the long axis of the NWs.

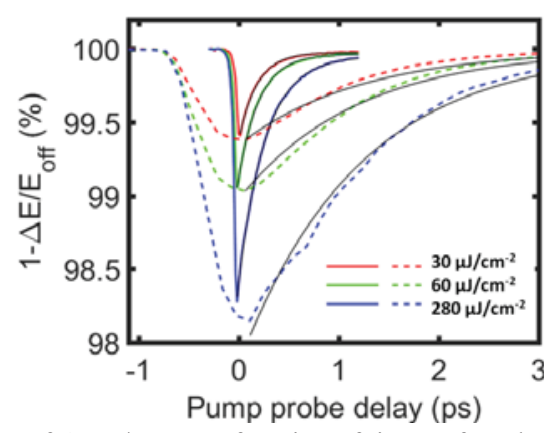

Figure 2. Measurements of $1-\Delta E / E_{\text {off }}$ as a function of time $t$ after photoexcitation at a range of pump fluences $\left(30-280 \mu \mathrm{J} \mathrm{cm}^{-2}\right.$ ). The extracted photoconductivity lifetime was $1.1 \pm 0.2 \mathrm{ps}$. Doted lines show data obtained using parylene films, solid colored lines show data obtained using PDMS films, black lines show fits. 
For all fluences, a sharp decrease in $1-\Delta E / E_{\text {off }}$ within 1 ps was observed followed by a rapid increase tending towards $100 \%$, attributed to trapping of free carriers at the GaAs NW surface. A monoexponential function was fitted to the rapid increase in the Parylene $\mathrm{C}$ data (Figure 2) to extract the photoconductivity lifetime, which was $1.1 \pm 0.2 \mathrm{ps}$ [7]. The polymer matrix did not make any significant difference to the magnitude of the modulation. The minimum of $\Delta E / E_{\text {off }}$ (at $\mathrm{t} \approx 0.01 \mathrm{ps}$ ) decreases with fluence because as photoexcitation fluence increases, the charge carrier density increases resulting in greater interaction with the $\mathrm{THz}$, resulting in a decrease in transmission. However, increasing the fluence eventually saturates 1 $\Delta E / E_{\text {off }}$ as conduction band filling occurs. This phenomenon is also attributed to a decrease in mobility as the excitation fluence increases.[15] This results in a limit on the modulation depth that can be achieved by increasing the pump fluence, therefore alternative device architectures were investigated.

In order to increase the modulation depth we increased the number of layers of polymernanowire thin films to fabricate the modulators. OPTP was used to characterize the modulator performance. The THz polarization was aligned to the long axis of the nanowires and the polarization of the photoexcitation beam was selected using a half waveplate. A rotation of the half waveplate by angle $\theta$ resulted in the rotation of the pump polarization by $2 \theta$. We measured $1-\Delta E / E_{\text {off }}$ with respect to $2 \theta$ to deduce the dependence of $\mathrm{THz}$ transmission with pump polarization. Multilayer samples were photoexcited at a fluence of $280 \mu \mathrm{J} \mathrm{cm}^{-2}$.

The thickness of the PDMS films and the mechanical instability of the laminated PDMS stacks made it difficult to fabricate multilayer samples consisting of more than 4 layers. Indeed, as shown in Figure 1b(iii) it is impossible to distinguish the nanowires through the PDMS due to the film thickness. In Figure 1b(ii) it is possible to distinguish domains where the nanowires were coated due to the thinness and conformal nature of the Parylene C.

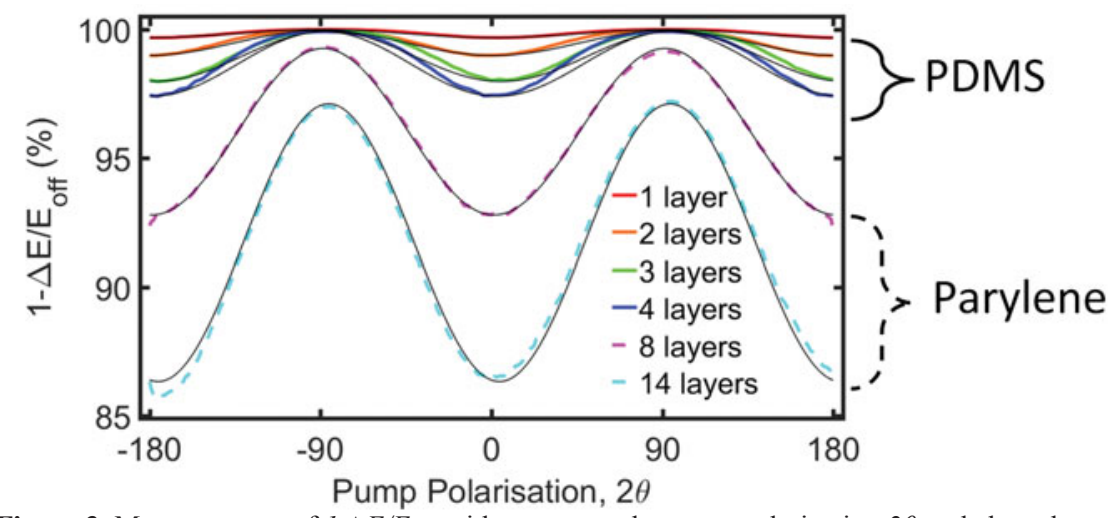

Figure 3. Measurements of $1-\Delta E / E_{\text {off }}$ with respect to the pump polarization $2 \theta$ to deduce the dependence of THz transmission with pump polarization (photoexcited at $280 \mu \mathrm{J} \mathrm{cm}^{-2}$ ) for (a) Parylene $\mathrm{C}$ and (b) PDMS. Modulation depth increased with number of layers, making Parylene $\mathrm{C}$ a better polymer matrix as multilayer samples up to 14 layers could be fabricated (only a maximum of 4 layers could be achieved using PDMS). Dotted lines show Parylene C. Data was taken at a pump time delay of zero. 
Compared to PDMS, it was possible to deposit much thinner films of Parylene C on the nanowires, and to remove these Parylene $\mathrm{C}$ films from the substrate.Therefore it was feasible to fabricate a 14 layer sample using Parylene $\mathrm{C}$.

For Figure 3, at $2 \theta=0^{\circ}$ and $2 \theta= \pm 180^{\circ}$, the nanowires are aligned with the polarization of the pump beam, resulting in photoexcited carriers being generated[16][17]. Maximum absorption of $\mathrm{THz}$ pulse occurs at these points as the THz pulse polarization is aligned with the pump polarization and nanowire orientation. As the pump polarization $2 \theta$ increases to $90^{\circ}$, the pump beam polarization becomes orthogonal to the nanowires and fewer charge carriers are produced. This results in transmission of the $\mathrm{THz}$ pulse. It is clear to see that by controlling the polarization of the photoexcitation pump beam, it is possible to modulate the transmission $\mathrm{THz}$ wave through the polymer-nanowire thin film. Fits to the data show a cosine-squared relationship in the THz transmission which is consistent with Malus's law for transmission through a polarizer[7]. As shown in Figure 3a and 3b, the modulation depth increases with the number of nanowire-polymer layers. We deduce that as the number of layers is increased, there is an increase in the effective areal density of nanowires, which results in the generation of more photoexcited carriers and therefore an increase in $\mathrm{THz}$ absorption. We extrapolate that layering as many films as possible could achieve a modulator with a high modulation depth. The more layers, the greater the density of aligned nanowires and photogenerated carriers, the more $\mathrm{THz}$ can be absorbed. The limitation of 14 layers was due to the amount of nanowire wafers available.

By measuring the photoexcited carrier lifetime using OPTP (described in Figure 1) and fitting a monoexponential function to the data in Figure 2 we found that the carrier lifetimes increased with the number of nanowire layers. The carrier lifetime is $1.1 \pm 0.2 \mathrm{ps}$ for the 1 layer sample and increases to $3.5 \pm 0.3 \mathrm{ps}$ for the 14 layer sample, which can be attributed to dispersion. This occurs when the refractive index of the nanowires and parylene at $\mathrm{THz}$ frequencies differs from that at the photoexcitation frequency. This causes the photoexcitation and $\mathrm{THz}$ pulses to propagate at slightly different speeds in the modulator, resulting in peak broading. We also note that dispersion effects in the Parylene samples are significantly lower than in the PDMS samples, as a single PDMS layer is 60 times thicker than a single Parylene layer. While the 14 layer samples had an increased photoexcitation lifetime, it is still on the picosecond scale, comparable to carbon nanotube $\mathrm{THz}$ modulators[7].

We found that the best performance modulator was the 14 layer device. Using OPTP measurements, we found that the THz transmission follows Malus's law and for the highest fluence of $280 \mu \mathrm{J} \mathrm{cm}^{-2}$, the extinction ratio was determined to be $13.5 \pm 1.2 \%$, on the way to being comparable to graphene $\mathrm{THz}$ modulators[6].

\section{CONCLUSIONS}

In summary, we demonstrate ultrafast THz modulators based on GaAs NWs embedded Parylene C and PDMS. We compared the performance of the two polymer matrices and found that due to the thinness of the Parylene $\mathrm{C}$ films and its suitability for hot bonding, several films can be layered together to fabricate the modulator. This dramatically increases the areal density of the nanowire, and therefore the $\mathrm{THz}$ absorption. We demonstrated a carrier lifetime of $3.5 \mathrm{ps}$ and a modulation depth of $13.5 \%$ from a 14 layer parylene-nanowire thin film modulator. This performance is comparable to carbon nanotube and graphene THz modulators. To further develop these modulators we suggestincreasing the number parylene-nanowire layers and finessing the NW growth process to improve NW alignment. 


\section{ACKNOWLEDGEMENTS}

The authors thank the EPSRC (U.K.) for financial support. H. J. Joyce thanks the Royal

Commission for the Exhibition of 1851 for her research fellowship.

\section{REFERENCES}

[1] M. Tonouchi, Nat. Photonics. 1, 97 (2007).

[2] M. B. Johnston, A. Dowd, R. Driver, E. H. Linfield, A. G. Davies, and D. M. Whittaker, Semicond. Sci. Technol.19, S449 (2004).

[3] K. Peng, P. Parkinson, L. Fu, Q. Gao, N. Jiang, Y. Guo, F. Wang, H. J. Joyce, J. L. Boland, H. H. Tan, C. Jagadish, and M. B. Johnston, Nano Lett. 15, 206 (2015).

4] T. Kleine-Ostmann and T. Nagatsuma, J Infrared Milli Terahz Waves. 32, 143 (2011).

[5] M. Rahm, J. Li, and W. J. Padilla, J Infrared Milli Terahz Waves, 34, 1 (2013).

[6] G. Liang, X. Hu, X. Yu, Y. Shen, L. H. Li, A. G. Davies, E. H. Linfield, H. K. Liang, Y. Zhang, S. F. Yu, and Q. J. Wang, ACS Photonics. 2, 1559 (2015).

[7] C. J. Docherty, S. D. Stranks, S. N. Habisreutinger, H. J. Joyce, L. M. Herz, R. J. Nicholas, and M. B. Johnston, J. Appl. Phys. 115, 13 (2014).

[8] H. J. Joyce, J. L. Boland, C. L. Davies, H. J. Joyce, C. J. Docherty, Q. Gao, and H. H. Tan, Nanotech. 7, 214006 (2013).

[9] H. J. Joyce, Q. Gao, H. H. Tan, C. Jagadish, Y. Kim, J. Zou, L. M. Smith, H. E. Jackson, J. M. Yarrison-Rice, P. Parkinson, and M. B. Johnston, Prog. Quantum Electron. 35, 23 ( 2011).

[10] P. Parkinson, H. J. Joyce, Q. Gao, H. H. Tan, X. Zhang, J. Zou, C. Jagadish, L. M. Herz, and M. B. Johnston, Nano Lett. 9, 1 (2009).

[11] P. Parkinson, J. Lloyd-hughes, Q. Gao, H. H. Tan, C. Jagadish, M. B. Johnston, and L. M. Herz, Nano Lett. 7, 2162 (2007).

[12] E. Meng, P.-Y. Li, and Y.-C. Tai, J. Micromech. Microeng. 18, 45004, (2008).

[13] H. Park, K. Seo, and K. B. Crozier, Appl. Phys. Lett. 101, 193107 (2012).

[14] H. Noh, K. Moon, A. Cannon, P. J. Hesketh, and C. P. Wong, J. Micromech. Microeng. 14, 625 (2004).

[15] H. J. Joyce, P. Parkinson, N. Jiang, C. J. Docherty, Q. Gao, H. H. Tan, C. Jagadish, L. M. Herz, and M. B. Johnston, Nano Letts. 14, 5989 (2014).

[16] H. J. Joyce, J. L. Boland, C. L. Davies, S. A. Baig, and M. B. Johnston, Semicond. Sci. Technol. 31,1 (2016).

[17] L. V Titova, T. B. Hoang, H. E. Jackson, L. M. S. M. Y. K. J. Joyce, H. H. Tan, C. Jagadish, L. V Titova, T. B. Hoang, H. E. Jackson, L. M. Smith, and Y. Kim, Appl. Phys. Lett., 1, 173126 (2006). 